\title{
Opciones terapéuticas en quistes odontogénicos. Revisión
}

\section{Therapeutic options in odontogenic cyst. Review}

\author{
Vega Llauradó A*, Ayuso Montero R**, Teixidor Olmo I, Salas Enric J***, \\ Marí Roig A****, López López J*****
}

\section{RESUMEN}

Los huesos maxilares constituyen asiento de una gran variedad de quistes y neoplasias que pueden ser de difícil diagnóstico. De entre todos los procesos tumorales que se dan en el territorio maxilofacial, los quistes son de gran importancia debido a la frecuencia de su presentación. Los quistes maxilares tienen distinto origen y comportamiento clínico. A partir de la clasificación de la OMS de 1992, esta revisión estudia las características clínicas, radiográficas y epidemiológicas de los quistes del desarrollo odontógenos. Una adecuada exploración clínica y radiográfica por parte del odontólogo es suficiente para alcanzar un diagnóstico de presunción. Las consideraciones clínicas y terapéuticas de cada uno de estos quistes son variables, por lo que es necesario conocer el comportamiento epidemiológico de ellos. El diagnóstico de presunción, el tamaño de la lesión y la relación de esta con estructuras anatómicas vecinas condicionará el tipo de tratamiento. El diagnóstico definitivo lo dictaminará el análisis anatomopatológico.

Palabras clave: Quiste odontogénico, quiste dentígero, quiste folicular, quiste de erupción, quiste periodontal lateral, quiste odontogénico glandular, queratoquiste, quiste primordial, tratamiento.

\section{SUMMARY}

The maxillary bones constitute the base of a big variety of cyst and tumours that can be difficult to diagnose. Among all the tumor processes that can occur in the maxillofacial area, cysts are of the utmost importance due to their frequency. Jaw cysts have different origins and clinical behavior. After the OMS classification in 1992, this review examines the clinical, radiographic and epidemiological characteristics of the cyst of the odontogenic development. An appropriate clinical and radiographic exploration on the part of the dentist is enough to reach a presumptive diagnosis. The clinical and therapeutic considerations of each of these cysts are variable, so it is necessary to know their epidemiological behavior. The presumptive diagnosis, the size of the cyst and its relationship with neighboring anatomical structures will determine the type of treatment. The definitive diagnosis will be determined by the anatomical and pathological diagnosis.

Key words: Odontogenic cyst, dentigerous cyst, follicular cyst, eruption cyst, lateral periodontal cyst, odontogenic glandular cyst, keratocyst, primordial cyst, treatment.

Fecha de recepción: 9 de abril de 2012.

Aceptado para publicación: 25 de abril de 2012.

Vega Llauradó A, Ayuso Montero R, Teixidor Olmo I, Salas Enric J, Marí Roig A, López López J. Opciones terapéuticas en quistes odontogénicos. Revisión. Av. Odontoestomatol 2013; 29 (2): 81-93.

* Odontólogo. Alumna del Máster en Medicina, Cirugía e Implantología Oral. Universidad de Barcelona.

** Doctor en Odontología. Profesor del Máster en Medicina, Cirugía e Implantología Oral. Universidad de Barcelona.

*** Doctor en Medicina y cirugía. Médico Estomatólogo. Profesor del Departamento de Odontoestomatología. Universidad de Barcelona.

**** Doctor en Medicina y cirugía. Cirujano Maxilofacial. Responsable de la Unidad Funcional de Cabeza y Cuello. Hospital Universitario de Bellvitge. 


\section{INTRODUCCIÓN}

Quiste se define como una bolsa conectivo-epitelial, tapizada en su interior por epitelio y recubierta en su cara externa por tejido conectivo, que encierra un contenido líquido o semilíquido. Son asintomáticos y se descubren en estudios radiográficos de rutina. Desde la primera clasificación propuesta por Robinson en 1945, se han sucedido múltiples clasificaciones. En esta revisión nos basamos en la clasificación de la OMS de 1992 (1) visible en la Tabla 1. De acuerdo a su patogénesis, se clasifican como lesiones del desarrollo o de tipo inflamatorio. Concretamente revisaremos los quistes del desarrollo clasificados como odontógenos. Debido a la dificultad diagnóstica que presentan los quistes de los maxilares, se realiza una revisión sobre el tema con el objetivo de clarificar la actitud diagnóstica y terapéutica para cada tipo de quiste. La Tabla 2 presenta los distintos tipos de quistes incluidos en la revisión junto con sus características clínicas, epidemiológicas y de tratamiento habituales. La práctica ha demostra-

\section{TABLA 1.- CLASIFICACIÓN DE LOS QUISTES DE LOS MAXILARES (Kramer, OMS 1992) (1)}

\section{A) Del desarrollo}

- Odontógenos:

- Quiste gingival infantil

- Quiste gingival adulto

- Queratoquiste (primordial)

— Quiste dentígero (folicular)

- Quiste de erupción

- Quiste periodontal lateral

- Quiste glandular

- Quiste odontogénico calcificante

- No odontógenos:

- Quiste nasolabial

- Quiste globulomaxilar

— Quiste nasopalatino

B) Inflamatorios

- Quiste radicular

- Quiste paradental

- Quiste residual do que existen dificultades en la interpretación clínica y radiológica de estas afecciones ya que muchas de ellas se asemejan entre sí. Debido a esto, se exponen criterios diagnósticos erróneos y conductas terapéuticas inadecuadas. Las consideraciones quirúrgicas de los quistes maxilares están relacionadas con los patrones clínicos e histológicos de cada uno de ellos. Todas estas consideraciones, junto con las ventajas e inconvenientes de cada tipo de tratamiento (Tabla 3) dictaminaran la opción terapéutica más adecuada.

\section{MATERIALES Y MÉTODOS}

Los artículos de la presente revisión se buscaron en noviembre de 2011, en la base de datos Pub Med, y se incluyeron los siguientes criterios de búsqueda: odontogenic cysts and treatment. La búsqueda se limitó a estudios en inglés, español y francés, a partir del año 2001 a 2011.

\section{RESULTADOS}

Fruto de esta combinación se obtuvieron 649 artículos. Se descartaron todos aquellos que no seguían la clasificación de Quistes de los maxilares de la OMS de 1992 y aquellos que trataban únicamente de quistes inflamatorios o tumores de los maxilares. De esta forma se obtuvieron 60 artículos que fueron analizados a texto completo. También se realizó una búsqueda manual en revistas específicas en español con los mismos criterios.

\section{DISCUSIÓN}

Plantearemos la discusión de los datos recogidos en base a dos aspectos, por un lado lo recogido en la literatura para cada una de las patologías y por otro lado bajo la perspectiva de las opciones terapéuticas en general.

\section{Tipos de quiste}

El diente deriva en parte del mesénquima (saco folicular, cemento, alveolo y ligamento), en parte del 
Vega Llauradó A, Ayuso Montero R, Teixidor Olmo I, Salas Enric J, Marí Roig A, López López J. Opciones terapéuticas en quistes odontogénicos. Revisión

\section{TABLA 2.- RESUMEN DE LAS CARACTERÍSTICAS CLÍNICAS, EPIDEMIOLÓGICAS Y TERAPÉUTICAS DE CADA TIPO DE QUISTE}

\begin{tabular}{|c|c|c|c|c|c|c|c|}
\hline Tipo de quiste & Frecuencia & Localización & Sexo & Edad & $\begin{array}{l}\text { Afectación } \\
\text { ósea }\end{array}$ & $\begin{array}{l}\text { Opciones } \\
\text { terapéuticas }\end{array}$ & Observaciones \\
\hline $\begin{array}{l}\text { Quiste gingival } \\
\text { del adulto }\end{array}$ & $\leq 0,5 \%$ & $\begin{array}{r}\text { Mandíbula } \\
(60-80 \%)\end{array}$ & Más en & $\begin{array}{c}5^{\mathrm{a}}-6^{\mathrm{a}} \\
\text { década }\end{array}$ & Infrecuente & - Biopsia excisional & $\begin{array}{l}\text { - Dientes asociados vitales } \\
\text { - Raramente es múltiple }\end{array}$ \\
\hline $\begin{array}{l}\text { Quiste } \\
\text { dentígero }\end{array}$ & $18-33 \%$ & $\begin{array}{l}3^{\circ} \mathrm{M} \text { inferior }> \\
\mathrm{C} \text { superior }> \\
3^{\circ} \mathrm{M} \text { superior }\end{array}$ & $\underset{\nearrow}{\text { Más en }}$ & $\begin{array}{c}2^{\mathrm{a}}-3^{\mathrm{a}} \\
\text { década }\end{array}$ & Si & $\begin{array}{l}\text { - Enucleación } \\
\text { - Descompresión } \\
\text { / Marsupialización }\end{array}$ & $\begin{array}{l}\text { - Se asocia a la corona de } \\
\text { dientes no erupcionados }\end{array}$ \\
\hline $\begin{array}{l}\text { Quiste de } \\
\text { erupción }\end{array}$ & $\leq 0,8 \%$ & $\begin{array}{l}\text { Más frecuente } \\
\text { en mandíbula }\end{array}$ & $\begin{array}{c}\text { Más en } \\
\sigma\end{array}$ & $\begin{array}{c}1^{\mathrm{a}} \\
\text { década }\end{array}$ & Infrecuente & $\begin{array}{l}\text { - Control y seguimiento } \\
\text { - Incisión liberadora } \\
\text { - Exéresis simple } \\
\text { - Marsupialización }\end{array}$ & $\begin{array}{l}\text { - Dentro de la mucosa } \\
\text { de un diente a punto } \\
\text { de erupcionar }\end{array}$ \\
\hline $\begin{array}{l}\text { Quiste } \\
\text { periodontal } \\
\text { lateral }\end{array}$ & $0,3-1,7 \%$ & Mandíbula & $\begin{array}{c}\text { Más en } \\
\sigma\end{array}$ & $\begin{array}{c}3^{a}-4^{a} \\
\text { década }\end{array}$ & Si & - Enucleación & $\begin{array}{l}\text { - Adherido a diente } \\
\text { erupcionado y vital }\end{array}$ \\
\hline Queratoquiste & $1-21 \%$ & $\begin{array}{l}\text { Mandíbula } \\
(65-80 \%)\end{array}$ & $\begin{array}{c}\text { Más en } \\
\sigma\end{array}$ & $\begin{array}{l}2^{\mathrm{a}}-3^{\mathrm{a}}-4^{\mathrm{a}} \\
\text { década }\end{array}$ & Si & $\begin{array}{l}\text { - Enucleación } \\
\text { - Enucleación }+ \\
\text { técnicas coadyuvantes } \\
\text { - Descompresión / } \\
\text { marsupialización } \\
\text { - Resección marginal } \\
\text { o en bloque }\end{array}$ & $\begin{array}{l}\text { - Alto índice de recurrencia } \\
\text { (20-30\%) } \\
\text { - Hasta un } 40 \% \text { de casos } \\
\text { asociados a un diente } \\
\text { no erupcionado }\end{array}$ \\
\hline
\end{tabular}

epitelio (lámina dentaria, ameloblastos y vaina de Hertwig) y en parte del ectomesénquima inducido por el epitelio (odontoblastos). A lo largo de la odontogénesis se forman una serie de estructuras que, tras cumplir su función, deben desaparecer. Sin embargo, pueden dejar tras de sí residuos o restos que pueden ser punto de partida de neoformaciones.

Tanto los quistes del desarrollo como los inflamatorios se caracterizan por un crecimiento lento y una tendencia expansiva. A pesar de ser entidades con un comportamiento benigno, pueden alcanzar un tamaño considerable si no se diagnostican y se tratan a tiempo (1-34).

A continuación se detallan las características clínicas y radiográficas, la epidemiología y las particularida- des de cada quiste de forma individual. El resumen de todas estas características aparece en la Tabla 2.

\section{Quiste Gingival del Adulto (QGA)}

Se trata de un quiste infrecuente, localizado típicamente en mandíbula (60-80\%), en la zona vestibular de caninos y premolares y aparece como una única y pequeña hinchazón gingival, de forma oval y con aspecto de contenido líquido. Suele aparecer entre la $5^{\mathrm{a}}-6^{\mathrm{a}}$ década de vida. Los dientes asociados son vitales. Raramente aparece de forma múltiple, ni unilateral ni bilateralmente, y tampoco suele aparecer en el vientre lingual. Al contrario que el Quiste Periodontal Lateral, el QGA es una lesión originada en la encía o la mucosa alveolar, no en el hueso 


\section{TABLA 3.- VENTAJAS E INCONVENIENTES DE LAS DIFERENTES OPCIONES TERAPÉUTICAS}

\begin{tabular}{|l|l|l|}
\hline Tipo de tratamiento & \multicolumn{1}{|c|}{ Ventajas } & \multicolumn{1}{c|}{ Inconvenientes } \\
\hline Enucleación (quistectomía) & - Técnica simple y habitual & - Alta tasa de recurrencias \\
\hline Descompresión/marsupialización & $\begin{array}{l}\text { - Permite disminuir el tamaño de la lesión } \\
\text { - Permite proteger estructuras anatómicas } \\
\text { vecinas al quiste } \\
\text { - En dentición temporal y gérmenes } \\
\text { no interfiere en la erupción de dientes } \\
\text { incluidos a causa del quiste }\end{array}$ & $\begin{array}{l}\text { - Requiere que el paciente sea } \\
\text { colaborador } \\
\text { - Muchas visitas de control } \\
\text { - Tratamiento de larga duración }\end{array}$ \\
\hline $\begin{array}{l}\text { Técnicas coadyuvantes } \\
\text { - Ostución de Carnoy } \\
\text { - Crioterapia } \\
\text { - Electrocauterización }\end{array}$ & $\begin{array}{l}\text { - Permite eliminar restos epiteliales en el hueso } \\
\text { adyacente al quiste }\end{array}$ & $\begin{array}{l}\text { - Disminuye las tasas de recurrencia } \\
\text { - Efectos cáusticos que pueden } \\
\text { dañar tejidos adyacentes }\end{array}$ \\
\hline Resección & - Mínima la tasa de recurrencia & $\begin{array}{l}\text { - Tratamiento muy agresivo } \\
\text { - Mayores complicaciones } \\
\text { postoperatorias } \\
\text { - Necesidad de reconstrucción } \\
\text { del defecto }\end{array}$ \\
\hline
\end{tabular}

alveolar. La afectación ósea es infrecuente, pero en ocasiones erosiona el hueso. La exposición radicular en el momento de la remoción del quiste es un hallazgo extremadamente raro (5-7).

La incidencia relativa del QGA en amplias series de casos se ha cifrado en $\leq 0,5 \%$ y tiene una ligera predilección por el sexo femenino (2-8). Entre los posibles diagnósticos diferenciales (DD) pueden incluirse: quiste periodontal lateral, fibroma, granuloma de células escamosas, granuloma piogénico, mucocele, quiste periapical de origen endodóntico y queratoquiste (5).

El tratamiento clásico del QGA es la biopsia excisional $(5,7)$.

\section{Quiste Dentígero (o folicular) (QD)}

Dicho quiste aparece como una lesión unilocular, radiolúcida, bien definida y asintomática (excepto cuando alcanza un tamaño considerable). Se asocia a la corona de dientes no erupcionados y clínicamente puede expandir el hueso cortical hasta tal punto que provoca el desplazamiento de los gérmenes y la reabsorción radicular de los dientes adyacentes (9-12). Suele aparecer en la $2^{a}-3^{a}$ década de vida $(9,10)$. Afecta más a hombres y aparece con más frecuencia asociado a los terceros molares inferiores, seguido por los caninos superiores y los terceros molares superiores Raramente afecta a dientes supernumerarios $(3,6,10,11)$. El QD se forma a partir del epitelio reducido del esmalte, y su contenido es líquido (11). La incidencia relativa del QD en amplias series de casos oscila entre el 18 y el 33\%, siendo el quiste odontogénico más frecuente por detrás del quiste radicular $(2,3,13)$.

Las opciones terapéuticas del QD son la descompresión, marsupialización o la enucleación según las características de las lesiones $(11,14)$. Los criterios que dictarán la modalidad de tratamiento son el tamaño y la localización de la lesión, la edad del paciente, la dentición que presenta y la posible afectación de estructuras anatómicas importantes (11). La exéresis simple estará indicada cuando no haya riesgo de lesionar estructuras anatómicas, tales como, ápices de dientes vitales vecinos, seno maxilar o el nervio dentario inferior. En cambio, la marsupializa- 
ción estará indicada cuando el quiste haya desplazado dientes, o si queremos que el diente impactado asociado al quiste erupcione (12).

\section{Quiste de Erupción (QE)}

El QE aparece clínicamente como una lesión de tejido blando, fluctuante, azulada e indolora. Se produce dentro de la mucosa que recubre un diente que está a punto de erupcionar, provocando una alteración en la erupción. En el pasado, se consideraba un tipo de quiste dentígero que ocurría en tejidos blandos. Raramente aparece en adultos. Su origen deriva del epitelio reducido del esmalte, aunque hay autores que sugieren que proviene de restos de la lámina dental (15).

La incidencia relativa del QE en amplias series de casos es $\leq 0,8 \%(3,6,8,13)$. Tiene ligera predilección por el sexo masculino, y se localiza con mayor frecuencia en la mandíbula. A diferencia de otros quistes, los exámenes radiográficos no son de utilidad en estos casos, ya que no suele haber afectación ósea (a diferencia del quiste dentígero).

Las opciones terapéuticas dependen de cada caso. Podemos no realizar ningún tratamiento y controlar, ayudar a erupcionar el diente mediante una incisión liberadora, realizar la exéresis o bien realizar una marsupialización. Por ejemplo, en casos de QE asociado a dientes natales, la exéresis estaría indicada cuando el diente natal tiene movilidad o escaso desarrollo (dado su mal pronóstico) o cuando interfiere en la alimentación del bebe. Aun así, la marsupialización es el tratamiento más habitualmente utilizado (15).

\section{Quiste Periodontal Lateral (QPL)}

Aparece por primera vez como entidad propia en la clasificación de los quistes maxilares de la OMS de 1992. Se trata de una lesión infrecuente, asintomática, que aparece adherida a la raíz de un diente erupcionado y vital $(16,17)$. Su incidencia relativa en amplias series de casos se sitúa entre el 0,3-1,7\%. Es más frecuente en hombres, su localización habitual es la mandíbula y suele aparecer en la $3^{\mathrm{a}}-4^{\mathrm{a}}$ década de vida $(2,3,6,8,13,18)$.
Histológicamente es una lesión radiolúcida con bordes bien definidos, uni o multilocular, adyacente a ápices de dientes vitales, tapizado por un fino epitelio plano estratificado no queratinizado. También presenta engrosamientos puntuales con células eosinofílicas ricas en glucógeno.

El tratamiento habitual es la enucleación del quiste, sin necesidad de realizar tratamiento endodóntico ni cirugía periapical del diente asociado, debido a su baja tasa de recurrencia $(16,17)$.

\section{Quiste Odontogénico Glandular (QOG)}

En el pasado se le llamaba quiste sialo-odontogénico, pero Gardner cambió su nombre por el de QOG, debido a la falta de evidencia sobre su origen en glándulas salivares. El quiste odontogénico glandular (QOG) es una lesión quística de los maxilares muy poco frecuente. De localización preferentemente mandibular (70-85\%). Radiológicamente aparece como una lesión radiolúcida, uni o multilocular, de bordes bien definidos y festoneados. De crecimiento lento, tiene una alta tasa de recurrencia $(\sim 25 \%)$ y un comportamiento localmente agresivo, pudiendo alcanzar grandes tamaños, erosionar las corticales o incluso provocar rizólisis. Aparece en un rango de edad amplio (con más frecuencia alrededor de los 50 años) (19-25). Sin predilección clara por ningún sexo, algunos autores afirman que afecta ligeramente más a mujeres (26) y otros a hombres $(19,25)$. Su incidencia relativa en amplias series de casos es $\leq 0,2 \%(3,6,8,13,27)$.

Histológicamente se trata de una cavidad quística tapizada por un epitelio plano estratificado no queratinizado, con células cuboidales y columnares, presentando áreas de engrosamiento, simulando pequeños quistes dentro del mismo. Estos también se presentan en el quiste periodontal lateral (19).

Entre los diagnósticos diferenciales del QOG hemos de incluir el QPL. Lo que le diferencia del QOG es la alta tasa de recurrencia de este último. El ameloblastoma y el granuloma de células gigantes también deben incluirse entre los DD.

El tratamiento adecuado es controvertido, pues los niveles de recurrencia tras la simple enucleación del 
quiste son elevados, y su comportamiento localmente agresivo fomenta un tratamiento quirúrgico más agresivo (osteotomía periférica o resección marginal) $(19,20)$. Kaplan y cols., (21) estudiaron la relación entre el tipo de tratamiento y la recidiva y observaron una importante reducción del número de recidivas en los casos tratados con osteotomía periférica o resección marginal. No hay suficiente evidencia que demuestre que tratamientos como la marsupialización, la criocirugía o las soluciones fijadoras tipo Carnoy reduzcan la incidencia de recurrencias. El riesgo de recurrencia es mayor si el QOG es de gran tamaño y multilocular (28).

\section{Queratoquiste (Quiste Primordial)}

El queratoquiste odontogénico también denominado quiste primordial es definido como un quiste del desarrollo de estirpe odontogénica. Se trata de una lesión benigna, pero localmente agresiva. En 2005, The World Health Organisation (WHO) lo reclasificó como "Tumor odontogénico queratoquístico (KCOT)" $(29,30)$. Su interés radica en su alto índice de recidivas, que se estima en un $20-30 \%$. Se presenta más frecuentemente en la segunda, tercera y cuarta décadas de la vida, con mayor incidencia en hombres que en mujeres, y con predilección por el ángulo y la rama mandibular (65-80\%) (29,31-36).

Radiográficamente pueden presentarse como lesiones uniloculares o multiloculares radiolúcidas de bordes nítidos, asociando una pieza dental no erupcionada hasta en un $40 \%$ de los casos. (Debe hacerse un diagnóstico diferencial con el quiste dentígero por este motivo). Pueden desplazar raíces de dientes adyacentes pero no se describen fenómenos de rizólisis. En caso de lesiones multiloculares es necesario descartar que se asocie a un Sd. de Gorlin $(31,33,36-38)$. Su incidencia relativa en amplias series de casos oscila entre el $1-21 \%(2,3,6,8,13,27)$. Otros autores sitúan su frecuencia entre $2-11 \%$ $(29,38)$.

La histología muestra una delgada pared de epitelio escamoso estratificado con células cuboideas o columnares en empalizada (5-10 capas), sin infiltrado inflamatorio. Estas células presentan habi- tualmente paraqueratosis, describiéndose también una minoría de casos con ortoqueratosis, los cuales presentarían un comportamiento menos agresivo, con menor tasa de recurrencia. El interior del quiste está relleno de un material cremoso de aspecto blanco sucio. También puede haber quistes satélite en el tejido conectivo de la pared del quiste $(31,34,37,39)$.

El tratamiento del queratoquiste abarca diversas opciones. La finalidad más importante es disminuir las altas tasas de recidiva de esta entidad, que pueden presentarse incluso diez años después de la cirugía. Las posibilidades de tratamiento incluyen la enucleación simple (no recomendada dadas las altas tasas de recidiva a las que se asocia: $17-56 \%$ ). Pueden emplearse técnicas coadyuvantes como la aplicación de solución de Carnoy tras la enucleación, o la crioterapia, disminuyendo así los índices de recidiva. Así mismo, se han empleado técnicas descompresivas derivadas de la marsupialización previas a la cirugía, con el fin de disminuir el tamaño del quiste y la presión intraluminal, permitiendo así una cirugía menos agresiva y con menores tasas de recidivas. En casos agresivos puede realizarse una resección mandibular en bloque $(29,38,39,40,41)$. Zecha y cols., (35) estudiaron los índices de recurrencia del KCOT durante un periodo de 35 años y concluyeron que el $21 \%$ de los KCOTs enucleados (sin tratamientos coadyuvantes) y el $40 \%$ de los KCOTs marsupializados recidivaban. Entre los factores de riesgo de recurrencia que se barajan está el acceso quirúrgico, la modalidad de tratamiento y la infección previa del quiste (42). En cambio, autores como Chirapathomsakul y cols., (36) no encuentran diferencias significativas entre las recurrencias y las diferentes modalidades de tratamiento.

\section{Opciones terapéuticas e indicaciones}

El tipo de tratamiento está condicionado por múltiples factores como el tamaño de la lesión, su localización, su relación con estructuras anatómicas vecinas, la posible afectación de estructuras dentales, entre otras (2). El objetivo es elegir la modalidad de tratamiento que conlleva el menor riesgo posible de recurrencia y la mínima morbilidad, y al mismo tiempo la erradicación de la lesión (41). 


\section{Enucleación (Quistectomía)}

La enucleación, tratamiento habitual de todos los quistes, consiste en la extirpación de una lesión despegándola del hueso. La eliminación completa del quiste permite el examen histopatológico de la lesión en su totalidad. En general, esta técnica quirúrgica va asociada a una alta tasa de recurrencia.

Muchos autores reportan dificultades en la enucleación y curetaje de queratoquistes con o sin perforación cortical debido a la adherencia al hueso o a los tejidos blandos adyacentes de la delgada capa que recubre el queratoquiste, especialmente en queratoquistes multiloculares y grandes. El revestimiento epitelial fino y friable, el acceso quirúrgico, la habilidad y experiencia del cirujano, la posible perforación cortical, y el deseo de preservar estructuras vitales adyacentes puede llevar a una extirpación incompleta del KCOT. Además, las lesiones recurrentes aparecen con mayor frecuencia en el área de la mandíbula y cuando el quiste va asociado a dientes incluidos, debido a la dificultad de acceso y por lo tanto incompleta remoción de todos los tejidos quísticos $(37,39,42,43)$. Para evitar este hecho, Sembronio y cols., (40) proponen ayudarse de la endoscopia para eliminar completamente un queratoquiste de grandes dimensiones. Aunque se ha demostrado que con tratamiento quirúrgico conservador puede resolverse la lesión preservando la anatomía y la función, Kolokythas y cols., (41) recuerdan que estos pacientes deben ser controlados durante muchos años, pues las recurrencias pueden aparecer pasados 10 años del tratamiento quirúrgico.

\section{Marsupialización y descompresión}

La marsupialización y la descompresión han sido utilizadas como métodos de tratamiento quirúrgico conservador en grandes quistes para minimizar el tamaño de estos, y por lo tanto limitar la extensión de la cirugía.

La marsupialización (Partsch I o quistotomía) fue descrita por primera vez por Partsch en 1892 para el tratamiento de las lesiones quísticas. Esta técnica se basa en la externalización de los quistes, a través de la creación de una ventana quirúrgica en la mucosa bucal y en la pared quística. Sus márgenes son suturados para crear una cavidad abierta que comunica con la cavidad oral. Este procedimiento alivia la presión del líquido del quiste, lo que permite la reducción del espacio quístico y facilita la aposición ósea en las paredes del quiste. La descompresión y la marsupialización son técnicas muy similares. La principal diferencia entre ellas reside en la creación de una ventana quirúrgica en la mucosa oral y de la membrana quística, y en el uso de un dispositivo cilíndrico (como la goma de un cuentagotas) o un drenaje quirúrgico rígido para evitar el cierre mucoso. El objetivo es mantener continua la comunicación entre la cavidad oral y el interior del quiste $(38,43-45)$. Este tratamiento requiere que el paciente sea colaborador, que irrigue el quiste regularmente y acuda a los controles. Por esta razón, sólo un selecto grupo de pacientes son candidatos para este tratamiento. El tiempo que dura la descompresión es uno de sus principales inconvenientes, pues el paciente pierde el interés en irrigarse correctamente la zona y en acudir a los controles periódicos (38). Además, en estos tratamientos prolongados, no es un hecho aislado el desprendimiento de las suturas y del drenaje que comunica la cavidad con el exterior (45).

Aunque hay múltiples estudios $(12,38,43,44,46)$ a largo plazo sobre la marsupialización, no está claro cómo de efectiva es a la hora de reducir el tamaño de las lesiones y cómo afecta a los índices de recurrencia (47), o qué mecanismos moleculares están implicados $(37,48)$. La marsupialización continúa siendo una técnica controvertida. Los porcentajes de recurrencia asociados a esta técnica que se encuentran en la literatura son muy dispares $(43,46,49)$. Tampoco hay consenso en la duración del tratamiento, es decir, en la cantidad de meses que la ventana quística debe permanecer abierta para que la reducción del quiste sea efectiva. En su serie de casos publicada, Nakamura y cols., (47) afirman que cuanto mayor es el tamaño del quiste, más efectiva es la marsupialización. Anavi y cols., (50) estudiaron 73 casos de quistes odontogénicos tratados con descompresión, y concluyeron que es un tratamiento válido para reducir el área de la lesión, pero que en casos de grandes lesiones, siempre es recomendable la remoción quirúrgica después de la descompresión. 
En el caso de los queratoquistes, Pogrel (44) y Pogreland Jordan (46) describen la utilización de marsupialización como tratamiento definitivo del KCOT. Estos últimos autores (46) describen 10 pacientes tratados exclusivamente con marsupialización y descompresión, logrando la resolución de las lesiones en todos los casos y con una tasa de recurrencia del $0 \%$. Jung y cols., (51) también recomiendan la descompresión en casos de grandes queratoquistes, porque a pesar de la controversia, estos tratamientos no conllevan las complicaciones postoperatorias que conlleva la cirugía radical.

La marsupialización y descompresión toman un cariz importante cuando se trata de quistes en niños con dentición decidua o mixta $(12,37)$. Los niños tienen mayor potencial que los adultos para regenerar hueso, y los dientes con ápices abiertos mayor potencial de erupción. Los quistes dentígeros asociados a un germen dentario o a un diente incluido son los más frecuentes en niños (3). El protocolo de tratamiento estándar para los quistes dentígeros es la enucleación y la extracción del diente afectado, pero, en pacientes infantiles, la marsupialización permite eliminar la lesión, la erupción de los dientes incluidos a causa del quiste, y no interfiere en el desarrollo de los gérmenes. Boj y cols., (10) recurren a técnicas con láser para abrir la ventana del quiste, aspirar su contenido y legrar, argumentando que el láser ofrece un efecto antibacteriano y antiinflamatorio que mejora el postoperatorio del paciente.

En pacientes ancianos, la marsupialización presenta ciertos inconvenientes: tratamiento muy largo, necesidad de higiene excelente (difícil en pacientes ancianos) y riesgo de infección de vías respiratorias.

\section{Tratamientos coadyuvantes}

De todas las terapias coadyuvantes que hay en la literatura, la solución de Carnoy, la osteotomía periférica, la crioterapia y la electrocauterización son las más comunes. El propósito de estos métodos es eliminar los posibles restos epiteliales de la pared quística que puedan quedar en el hueso adyacente y que pueden inducir a recurrencias $(30,37,52)$. La solución Carnoy (compuesta de alcohol absoluto: 6 ml, cloroformo: $3 \mathrm{ml}$, ácido acético: $1 \mathrm{ml}$, y cloruro férrico: $1 \mathrm{~g}$ ) tiene una propiedad quelante con moderada penetración en el hueso y una fijación local rápida. Así como excelente hemostasia.

En el caso de los queratoquistes, Morgan y cols., (53) afirman que la osteotomía periférica (con o sin aplicación de solución de Carnoy) disminuye notablemente los índices de recurrencia. Otros autores como Tolstunov (54), opinan que la osteotomía periférica puede dispersar los microquistes que están incrustados en el hueso adyacente, aumentando así el riesgo de recurrencias. Stoelinga y cols., (55) afirman que en el caso de los queratoquistes multiloculares, es necesario eliminar los septos intralesionales antes de la enucleación y/o curetaje de la lesión, y la aplicación de solución de Carnoy, y que esta técnica reduce los índices de recurrencia (en comparación con la enucleación simple) y la morbilidad (en comparación con la resección). Para Stoelinga (55), 5 minutos de aplicación de la solución de Carnoy son suficientes para eliminar los restos epiteliales remanentes, mientras que Zhao y cols., (56) la aplican durante 10-15 minutos (excepto cuando hay riesgo de afectación nerviosa, que lo aplican $\leq 3$ minutos). Sin embargo, los principales inconvenientes de la solución de Carnoy son su efecto cáustico que puede dañar los tejidos adyacentes y nerviosos (56). Para Stoelinga (57), la electrocauterización es probablemente más eficaz para eliminar los restos epiteliales porque su efecto cauterizador es más profundo que el de la solución de Carnoy.

Por otro lado, Tonietto y cols., (52) abogan por el uso de nitrógeno líquido como tratamiento coadyuvante, porque afirman que el nitrógeno líquido preserva las propiedades inorgánicas del hueso, mientras que la solución de Carnoy destruye las propiedades osteogénicas y osteoconductivas del hueso. Schmidt y cols., (58) también apuestan por combinar la enucleación con crioterapia con nitrógeno líquido dados los buenos resultados que obtienen. En cambio Madras y cols., (29) refieren que la enucleación con crioterapia no ofrece ninguna mejora y en cambio la enucleación con solución de Carnoy sí que reduce de forma significativa las recurrencias. Stoelinga y cols., (55) opina que la crioterapia con nitrógeno líquido es un método más complejo que la solución de Carnoy y potencialmente lesivo para 
los tejidos subyacentes, como el nervio dentario inferior.

En el caso de los Quistes Odontogénicos Glandulares, Qin y cols., (59) recomiendan la enucleación más fijación con solución de Carnoy como el método más adecuado. En cuanto a los QOG de grandes dimensiones y multiloculares, Kaplan y cols., (20) recomiendan la osteotomía periférica, o incluso la resección, debido a las altas recurrencias de estos quistes.

\section{Resección}

La resección radical es una técnica más amplia en la que se incluye en la extirpación del quiste un área ósea alrededor del mismo como margen quirúrgico, tratando de eliminar todo el tejido epitelial que puede existir en la periferia del quiste con el fin de prevenir la recidiva. Dependiendo del tamaño y localización del quiste, la resección puede ser marginal (preservando la basal ósea) o segmentaria (si se trata de grandes quistes en los que el margen óseo sano sea escaso y no permita preservar la continuidad ósea maxilar o mandibular). La resección segmentaria debe seguirse de la reconstrucción del defecto de acuerdo con los métodos habituales. Aunque se ha descrito menor tasa de recidiva en casos de resección quirúrgica frente a los otros métodos discutidos (marsupialización, enucleación o enucleación asociada a terapias coadyuvantes), la resección segmentaria puede también presentar recurrencia, incluso se ha descrito recurrencia en el injerto óseo usado para la reparación del defecto (36). La resección radical es por tanto controvertida.

Basándose en los elevados índices de recurrencia de los KCOTs y los QOG, algunos abogan por la enucleación (con o sin terapias coadyuvantes) de los pequeños quistes uniloculares, y sugieren la resección (marginal o segmentaria) y el injerto en los casos de grandes lesiones $(20,43)$. Aunque en el pasado, autores como Worrall (60) recomendaban la resección como el tratamiento de elección para KCOTs que han erosionado hueso, otros como Zhao (56) consideran que la resección debe tenerse especialmente en cuenta para el tratamiento de un KCOT recurrente, en cuyo caso debe extenderse más allá de la mayor extensión de la lesión para asegurar la eliminación completa de restos de los quistes satélites o restos epiteliales de la pared del quiste.

Aunque los niveles de recurrencia después de una resección radical son nulos o muy bajos, las complicaciones postoperatorias y los defectos que deja en el paciente son muy agresivos, y no están justificados tratándose de una patología benigna (43). Las complicaciones más frecuentes de la cirugía resectiva de los queratoquistes de los maxilares son la infección del lecho quirúrgico, la lesión del nervio alveolar inferior en quistes mandibulares y la fractura patológica.

\section{CONCLUSIONES}

Los quistes odontogénicos son entidades con un comportamiento benigno, pero si no se diagnostican a tiempo pueden alcanzar tamaños considerables y repercutir negativamente en el paciente.

En el momento en que una lesión se diagnostica como compatible con quiste, ya sea clínicamente o radiográficamente, se debe establecer un diagnóstico diferencial y de presunción.

Las características clínicas, radiográficas, así como la epidemiología, son las principales herramientas disponibles para hacer un diagnóstico de presunción.

El diagnóstico de presunción, el tamaño de la lesión y la relación de esta con estructuras anatómicas vecinas condicionará el tipo de tratamiento.

El diagnóstico definitivo lo dictaminará el análisis anatomopatológico.

\section{BIBLIOGRAFÍA}

1. Kramer IR, Pindborg JJ, Shear M. The WHO Histological Typing of OdontogenicTumours. A commentary on the Second Edition. Cancer 1992;70(12):2988-94

2. Núñez-Urrutia S, Figueiredo R, Gay-Escoda C. Retrospective clinicopathological study of 418 
odontogenic cysts. Med Oral Patol Oral Cir Bucal 2010;15(5):e767-3.

3. Ochsenius G, Escobar E, Godoy L, Peñafiel C. Odontogenic cysts: analysis of 2,944 cases in Chile. Med Oral Patol Oral Cir Bucal 2007;12(2):e85-91.

4. Koseoglu BG, Atalay B, Erdem MA. Odontogenic cysts: a clinical study of 90 cases. J Oral Sci 2004;46(4):253-7.

5. Kelsey WP 5th, Kalmar JR, Tatakis DN. Gingival cyst of the adult: regenerative therapy of associated root exposure. A case report and literature review. J Periodontol 2009;80(12):2073-81.

6. Jones AV, Craig GT, Franklin CD. Range and demographics of odontogenic cysts diagnosed in a UK population over a 30-year period. J Oral Pathol Med 2006; 35(8):500-7

7. Giunta JL. Gingival cysts in the adult. J Periodontol 2002;73(7):827-31.

8. Grossmann SM, Machado VC, Xavier GM, Moura MD, Gómez RS, Aguiar MC, Mesquita RA. Demographic profile of odontogenic and selected nonodontogenic cysts in a Brazilian population. Oral Surg Oral Med Oral Pathol Oral Radiol Endod 2007;104(6):e35-41.

9. Hu YH, Chang YL, Tsai A. Conservative treatment of dentigerous cyst associated with primary teeth. Oral Surg Oral Med Oral Pathol Oral RadiolEndod 2011;112(6):e5-7

10. Boj JR, Poirier C, Hernandez M, Espasa E. Laserassisted treatment of a dentigerous cyst: case report. Pediatr Dent 2007;29(6):521-4.

11. Motamedi MH, Talesh KT. Management of extensive dentigerous cysts. Br Dent J 2005;198 (4):203-6.

12. Ertas U, Yavuz MS. Interesting eruption of 4 teeth associated with a large dentigerous cyst in mandible by only marsupialization. J Oral MaxillofacSurg 2003;61(6):728-30.
13. Mosqueda-Taylor A, Irigoyen-Camacho ME, DiazFranco MA, Torres-Tejero MA. Odontogenic cysts. Analysis of 856 cases. Med Oral 2002;7(2):89-96.

14. Cakarer S, Selvi F, Isler SC, Keskin C. Decompression, enucleation, and implant placement in the management of a large dentigerous cyst. J Craniofac Surg 2011;22(3): 922-4.

15. Bodner L, Goldstein J, Sarnat H. Eruption cysts: a clinical report of 24 new cases. J ClinPediatr Dent 2004;28(2):183-6.

16. Formoso Senande MF, Figueiredo R, Berini Aytés L, Gay Escoda C. Lateral periodontal cysts: a retrospective study of 11 cases. Med Oral Patol Oral Cir Bucal2008;13(5):E313-7.

17. Nart J, Gagari E, Kahn MA, Griffin TJ. Use of guided tissue regeneration in the treatment of a lateral periodontal cyst with a 7-month reentry. J Periodontol 2007;78(7):1360-4.

18. de Souza LB, Gordón-Núñez MA, Nonaka CF, de Medeiros MC, Torres TF, Emiliano GB. Odontogenic cysts: demographic profile in a Brazilian population over a 38-year period. Med Oral Patol Oral Cir Bucal 2010;15(4):e583-90.

19. Krishnamurthy A, Sherlin HJ, Ramalingam K, Natesan A, Premkumar P, Ramani P, Chandrasekar T. Glandular odontogenic cyst: report of two cases and review of literature. Head Neck Pathol 2009;3(2):153-8.

20. Kaplan I, Anavi Y, Hirshberg A. Glandular odontogenic cyst: a challenge in diagnosis and treatment. Oral Dis 2008;14(7):575-81.

21. Kaplan I, Gal G, Anavi Y, Manor R, Calderon S. Glandular odontogenic cyst: treatment and recurrence. J Oral Maxillofac Surg 2005;63(4): 435-41.

22. Boffano P, Cassarino E, Zavattero E, Campisi P, Garzino-Demo P. Surgical treatment of glandular odontogenic cysts. J Craniofac Surg 2010; 21(3): 776-80. 
23. Lyrio MC, de Assis AF, Germano AR, de Moraes M. Treatment of mandibular glandular odontogenic cyst with immediate reconstruction: case report and 5-year follow-up. Br J Oral Maxillofac Surg 2010;48(8):651-3.

24. Macdonald-Jankowski DS. Glandular odontogenic cyst: systematic review. Dentomaxillofac Radiol 2010;39(3):127-39.

25. Noffke C, Raubenheimer EJ. The glandular odontogenic cyst: clinical and radiological features; review of the literature and report of nine cases. Dentomaxillofac Radiol 2002;31 (6):333-8.

26. Thor A, Warfvinge G, Fernandes R. The course of a long-standing glandular odontogenic cyst: marginal resection and reconstruction with particulated bone graft, platelet-rich plasma, and additional vertical alveolar distraction. J Oral Maxillofac Surg 2006;64(7):1121-8.

27. Meningaud JP, Oprean N, Pitak-Arnnop P, Bertrand JC. Odontogenic cysts: a clinical study of 695 cases. J Oral Sci 2006;48(2):59-62.

28. Ninomiya T, Kubota Y, Koji T, Shirasuna K. Marsupialization inhibits interleukin-1alpha expression and epithelial cell proliferation in odontogenic keratocysts. J Oral Pathol Med 2002; 31(9):526-33.

29. Madras J, Lapointe H. Keratocystic odontogenic tumour: reclassification of the odontogenic keratocyst from cyst to tumour. J Can Dent Assoc 2008;74(2):165-5.

30. Sharif FNj, Oliver R, Sweet C, Sharif MO. Interventions for the treatment of keratocystic odontogenic tumours (KCOT, odontogenic keratocysts (OKC)). Cochrane Database Syst Rev 2010;8(9):CD008464.

31. O'Neill R, Al-Hezaimi K. Identification of an odontogenic keratocyst and treatment with guided tissue regeneration: case report. J Can Dent Assoc 2011;77:b6
32. González-Alva P, Tanaka A, Oku Y, Yoshizawa D, Itoh S, Sakashita H, Ide F, Tajima Y, Kusama K. Keratocystic odontogenic tumor: a retrospective study of 183 cases. J Oral Sci 2008;50(2):205-12.

33. Habibi A, Saghravanian N, Habibi M, Mellati E, Habibi M. Keratocystic odontogenic tumor: a 10year retrospective study of 83 cases in an Iranian population. J Oral Sci 2007;49(3):229-35.

34. Ruhin-Poncet B, Picard A, Martin-Duverneuil N, Albertini AF, Goudot P. Keratocysts (or keratocystic epithelial odontogenic tumors). Rev Stomatol Chir Maxillofac 2011;112(2):87-92.

35. Zecha JA, Mendes RA, Lindeboom VB, van der Waal I. Recurrence rate of keratocystic odontogenic tumor after conservative surgical treatment without adjunctive therapies - A 35-year single institution experience. Oral Oncol 2010;46 (10):740-2.

36. Chirapathomsakul D, Sastravaha P, Jansisyanont P. A review of odontogenic keratocysts and the behavior of recurrences. Oral Surg Oral Med Oral Pathol Oral Radiol Endod 2006;101(1):5-9.

37. Mendes RA, Carvalho JF, van der Waal I. Characterization and management of the keratocystic odontogenic tumor in relation to its histopathological and biological features. Oral Oncol 2010;46(4):219-25.

38. Maurette PE, Jorge J, de Moraes M. Conservative treatment protocol of odontogenic keratocyst: a preliminary study. J Oral Maxillofac Surg 2006; 64(3):379-83.

39. Pitak-Arnnop P, Chaine A, Oprean N, Dhanuthai $\mathrm{K}$, Bertrand JC, Bertolus C. Management of odontogenic keratocysts of the jaws: a ten-year experience with 120 consecutive lesions. J Craniomaxillofac Surg 2010;38(5):358-64.

40. Sembronio S, Albiero AM, Zerman N, Costa F, Politi $M$. Endoscopically assisted enucleation and curettage of large mandibular odontogenic keratocyst. Oral Surg Oral Med Oral Pathol Oral Radiol Endod 2009;107(2):193-6. 
41. Kolokythas A, Fernandes RP, Pazoki A, Ord RA. Odontogenic keratocyst: to decompress or not to decompress? A comparative study of decompression and enucleation versus resection/ peripheral ostectomy. J Oral Maxillofac Surg 2007;65(4):640-4.

42. Chemli H, Dhouib M, Karray F, Abdelmoula M. Risk factors for recurrence of maxillary odontogenic keratocysts. Rev Stomatol Chir Maxillofac 2010;111(4):189-92.

43. Giuliani M, Grossi GB, Lajolo C, Bisceglia M, Herb KE. Conservative management of a large odontogenic keratocyst: report of a case and review of the literature. J Oral Maxillofac Surg 2006;64(2):308-16.

44. Pogrel MA. Treatment of keratocysts: the case for decompression and marsupialization. J Oral Maxillofac Surg 2005;63(11):1667-73.

45. Swantek JJ, Reyes MI, Grannum RI, Ogle OE. A Technique for Long Term Decompression of Large Mandibular Cysts. J Oral Maxillofac Surg 2012;70(4):856-9.

46. Pogrel MA, Jordan RC. Marsupialization as a definitive treatment for the odontogenic keratocyst. J Oral Maxillofac Surg 2004;62(6): 651-5.

47. Nakamura N, Mitsuyasu T, Mitsuyasu Y, Taketomi T, Higuchi Y, Ohishi M. Marsupialization for odontogenic keratocysts: long-term follow-up analysis of the effects and changes in growth characteristics. Oral Surg Oral Med Oral Pathol Oral RadiolEndod 2002; 94(5):543-53.

48. Mendes RA, Carvalho JF, van der Waal I. Biological pathways involved in the aggressive behavior of the keratocystic odontogenic tumor and possible implications for molecular oriented treatment - an overview. Oral Oncol 2010;46(1): $19-24$.

49. Myoung H, Hong SP, Hong SD, Lee JI, Lim CY, Choung PH, Lee JH, Choi JY, Seo BM, Kim MJ. Odontogenic keratocyst: Review of 256 cases for recurrence and clinicopathologic parameters. Oral Surg Oral Med Oral Pathol Oral Radiol Endod 2001;91(3):328-33.

50. Anavi Y, Gal G, Miron H, Calderon S, Allon DM. Decompression of odontogenic cystic lesions: clinical long-term study of 73 cases. Oral Surg Oral Med Oral Pathol Oral Radiol Endod 2011; 112(2):164-9.

51. Jung YS, Lee SH, Park HS. Decompression of large odontogenic keratocysts of the mandible. J Oral Maxillofac Surg 2005; 63(2):267-71.

52. Tonietto L, Borges HO, Martins CA, Silva DN, Sant'Ana Filho M. Enucleation and liquid nitrogen cryotherapy in the treatment of keratocystic odontogenic tumors: a case series. J Oral Maxillofac Surg 2011;69(6):e112-7.

53. Morgan TA, Burton CC, Qian F. A retrospective review of treatment of the odontogenic keratocyst. J Oral MaxillofacSurg 2005;63(5): 635-9.

54. Tolstunov L, Treasure T. Surgical treatment algorithm for odontogenic keratocyst: combined treatment of odontogenic keratocyst and mandibular defect with marsupialization, enucleation, iliac crest bone graft, and dental implants. J Oral Maxillofac Surg 2008;66(5):1025-36.

55. Stoelinga PJ. The treatment of odontogenic keratocysts by excision of the overlying, attached mucosa, enucleation, and treatment of the bony defect with carnoy solution. J Oral Maxillofac Surg 2005;63(11):1662-6.

56. Zhao YF, Wei JX, Wang SP. Treatment of odontogenic keratocysts: a follow-up of 255 Chinese patients. Oral Surg Oral Med Oral Pathol Oral Radiol Endod 2002;94(2):151-6.

57. Stoelinga PJ. Long-term follow-up on keratocysts treated according to a defined protocol. Int J Oral Maxillofac Surg 2001;30(1):14-25.

58. Schmidt BL, Pogrel MA. The use of enucleation and liquid nitrogen cryotherapy in the 
management of odontogenic keratocysts. J Oral Maxillofac Surg 2001;59(7):720-5.

59. Qin XN, Li JR, Chen XM, Long X. The glandular odontogenic cyst: clinicopathologic features and treatment of 14 cases. J Oral Maxillofac Surg 2005;63(5):694-9.

60. Worrall SF. Recurrent odontogenic keratocyst within the temporalis muscle. Br J Oral Maxillofac Surg 1992;30(1):59-62.

\section{CORRESPONDENCIA}

José López López

Campus Universitario de Bellvitge Departamento de Odontoestomatología Pabellón de Gobiereno, $2^{a}$ planta Feixa Llarga, s/n 08907 L'Hospitalet de Llobregat Barcelona

18575jll@gmail.com 